\title{
A Note on the Stability of an Iterative Finite- Difference Method for Hyperbolic Systems
}

\author{
By Moshe Goldberg
}

Abstract. In this note, we find analytically the linear stability criteria for two finitedifference methods for hyperbolic systems in conservation-law form, presented recently by S. Abarbanel and G. Zwas and by S. Abarbanel and M. Goldberg.

Introduction. Consider the first-order quasi-linear hyperbolic system of partial differential equations

$$
\partial W / \partial t+A \partial W / \partial x=0, \quad-\infty<x<\infty, t \geqq 0 .
$$

$W=\left(W_{1}, \cdots, W_{m}\right)$ is the vector of the unknowns and $A=A\left(W_{1}, \cdots, W_{m}\right)$ is a square matrix. Hyperbolicity implies that the eigenvalues of $A$ are real, and we assume that the system is in a conservation-law form, i.e., $A \partial W / \partial x=\partial F / \partial x$ where $F=$ $F\left(W_{1}, \cdots, W_{m}\right)$ is a vector. Hence, Eq. (1) takes the form

$$
\partial W / \partial t=-\partial F / \partial x \text {. }
$$

The Lax-Wendroff finite-difference approximation [3] for (1a) is

$$
\begin{aligned}
W_{i}^{n+1}= & W_{i}^{n}-(\lambda / 2)\left[F_{i+1}^{n}-F_{i-1}^{n}\right] \\
& +\left(\lambda^{2} / 2\right)\left[A_{i+1 / 2}^{n}\left(F_{i+1}^{n}-F_{j}^{n}\right)-A_{i-1 / 2}^{n}\left(F_{i}^{n}-F_{i-1}^{n}\right)\right],
\end{aligned}
$$

where $f_{i}^{n}=f\left(x_{i}, t_{n}\right), A_{i \pm 1 / 2}^{n}=\frac{1}{2}\left(A_{i}^{n}+A_{i \pm 1}^{n}\right)$ and $\lambda=\Delta t / \Delta x$. Writing Eq. (2) in the form

$$
W_{i}^{n+1}=W_{i}^{n}+Q W_{i}^{n},
$$

Abarbanel and Zwas [1] presented the following iterative scheme:

$$
W_{i}^{n+1, s+1}=W_{i}^{n}+Q\left[\theta W_{i}^{n+1, s}+(1-\theta) W_{i}^{n}\right], \quad \frac{1}{2} \leqq \theta \leqq 1,
$$

where $s=0,1, \cdots, l-1$ and $W_{i}^{n+1,0} \equiv W_{i}^{n}$. For $\theta=0$ or $l=0$ (no iterations), we go back to (2); for $\theta \neq 0$ and $l \geqq 1$, we get a first-order accurate scheme. Another version of (3) was given by Abarbanel and Goldberg [2]:

$$
W_{i}^{n+1, s+1}=W_{i}^{n}+\theta Q W_{i}^{n+1, s}+(1-\theta) Q W_{i}^{n}, \quad \theta \text { real. }
$$

Since usually $Q$ is not a linear operator, (3) and (3a) in general do not coincide for $\theta \neq 1$, and they were referred to, respectively, as the "internal" and the "external" schemes.

Numerical experiments (see [2] for example) give evidence that the external scheme is much more dissipative when it comes to shock-containing problems and a first-

Received February 22, 1972, revised March 22, 1972.

AMS (MOS) subject classifications (1970). Primary 65M10.

Key words and phrases. Difference methods, stability. 
order accurate scheme is desired. However, a general observation of importance is that one iteration $(l=2)$ suffices to smooth out the numerical noise and, as a matter of fact, increasing $l$ causes in the beginning worsening of the phenomenon. In [1], linear stability analysis was done only for $\frac{1}{2} \leqq \theta \leqq 1$, and was carried out mostly numerically (except for specific values of $\theta$, for which it was done analytically). In [2], the stability analysis was carried out for all real $\theta$, but still in the same manner. Generally, it was found that the smoothest results occur in a region $\theta \geqq \theta_{0}$ where $\theta_{0} \simeq$ 0.166. For $0<\theta<\theta_{0}$, numerical solutions were not free of nonlinear oscillations. In this note, we derive analytically the following linear stability conditions of (3) and (3a) for the first iteration, namely for $l=2$ :

$$
\lambda \rho(A) \leqq 1 / \sqrt{2 \theta} \text { for } \theta \geqq 1 / 6,
$$

where $\rho(A)$ is the spectral radius of $A$. In addition, we show that the schemes are unconditionally unstable for $\theta<0$.

Stability Analysis. We are now ready to derive stability conditions for the linearized versions of (3) and (3a) which are identical, since we take $A$ to be locally constant, thus making $Q$ linear.

Of course, when the iterative schemes (3) and (3a) are applied to situations which include discontinuities, the linear stability cannot predict nonlinear instabilities. We just note that in all known schemes, such as Lax-Wendroff, etc., only linear stability is usually investigated. Nonlinear instabilities are combated in various ways, often through the introduction of artificial viscosity. However, the stabilizing effect of artificial viscosity, for example, is also investigated linearly. The iterative schemes which are analyzed here do away with the need to use a stabilizing term such as artificial viscosity.

Denoting the final result $W_{i}^{n+1,2}$ by $W_{i}^{n+1}$ and the linearized version of $Q$ by $P$, we get (see (3a))

$$
W_{i}^{n+1}=W_{i}^{n}+(1-\theta) P W_{i}^{n}+\theta P\left(W_{i}^{n}+P W_{i}^{n}\right)=\left(I+P+\theta P^{2}\right) W_{i}^{n},
$$

where (see (2))

$$
P W_{i}^{n}=-(\lambda / 2) A\left(W_{j+1}^{n}-W_{i-1}^{n}\right)+\left(\lambda^{2} / 2\right) A^{2}\left(W_{j+1}^{n}-2 W_{i}+W_{j-1}^{n}\right) .
$$

Taking the Fourier transform of (4) by setting $W_{i}^{n}=T_{n} \exp \left[i k x_{j}\right]$, Eq. (4) takes the form

$$
W_{i}^{n+1}=G W_{i}^{n},
$$

where the amplification matrix $G$ is given by

$$
\begin{aligned}
G= & I-i \lambda \sin \alpha \cdot A-\lambda^{2} A^{2}(1-\cos \alpha) \\
& +\theta\left[i \lambda \sin \alpha A+\lambda^{2} A^{2}(1-\cos \alpha)\right]^{2}, \quad \alpha=k \Delta x .
\end{aligned}
$$

Hence, the corresponding eigenvalues of $G$, say $g$, are

$$
g=1-i \lambda a \sin \alpha-\lambda^{2} a^{2}(1-\cos \alpha)+\theta\left[i a \lambda \sin \alpha+\lambda^{2} a^{2}(1-\cos \alpha)\right]^{2},
$$

where the $a$ 's are the eigenvalues of $A$. Following von Neumann, we would like to find the conditions under which 


$$
|g|^{2} \leqq 1
$$

Setting $b=\lambda^{2} a^{2}, \omega=\sin ^{2} \alpha / 2$, we have from (6)

$$
\begin{aligned}
|g|^{2}= & 1-4 b \omega \\
& \cdot\left[\omega-b \omega+2 \theta\left(1-\omega+2 b^{2} \omega^{2}+b \omega-2 b \omega^{2}\right)-4 \theta^{2} b \omega(1-\omega+b \omega)^{2}\right] .
\end{aligned}
$$

Since $b$ and $\omega$ are nonnegative, our aim is to find the largest $b_{\max }$ such that, for all $0 \leqq b \leqq b_{\max }$, the expression in the square brackets in $(8 \mathrm{a})$, which will be denoted by $R(b, \omega)$, will satisfy the relation $R(b, \omega) \geqq 0$ uniformly in $\omega(0 \leqq \omega \leqq 1)$. Namely, we are looking for the largest rectangle in the $(b, \omega)$ plane with vertices $(0,0),\left(b_{\max }, 0\right)$, $(0,1),\left(b_{\max }, 1\right)$ in which $R(b, \omega) \geqq 0$. This will lead us to a stability condition of the form

$$
\lambda^{2} a^{2} \leqq b_{\max } .
$$

Consider the following transformation of the $(b, \omega)$ plane:

$$
\xi=1-\omega+b \omega, \quad \eta=2 b \omega .
$$

For fixed $\omega_{0} \neq 0$ and $b_{0}$, the lines $\left(b, \omega_{0}\right)$ and $\left(b_{0}, \omega\right)$ in the $(b, \omega)$ plane are transferred, respectively, to the lines $(\xi, \eta)=\left(1-\omega_{0}+b \omega_{0}, 2 \omega_{0} b\right)$ and $(\xi, \eta)=(1-$ $\left.\omega+b_{0} \omega, 2 \omega b_{0}\right)$ in the $(\xi, \eta)$ plane, so that the singularity of this mapping is reflected through the fact that the image of the line $(0, b)$ is the point $(\xi, \eta)=(1,0)$. Therefore, for our purpose, this transformation is still valid because $|g|^{2}=1$ for $\omega=0$. Now, we conclude that the image of the half-infinite strip $\{(b, \omega) \mid b \geqq 0,0 \leqq \omega \leqq 1\}$ is the half-infinite strip $\{(\xi, \eta) \mid 0 \leqq \eta \leqq 2 \xi, \eta>2 \xi-2\}$, and each line $b=b_{0}$ parallel to the $\omega$-axis, is transferred to the line $\eta=\left[2 b_{0} /\left(1-b_{0}\right)\right](1-\xi)$ that passes through the point $(1,0)$ in the $(\xi, \eta)$ plane (see Fig. 1). Under the above-mentioned transforma-

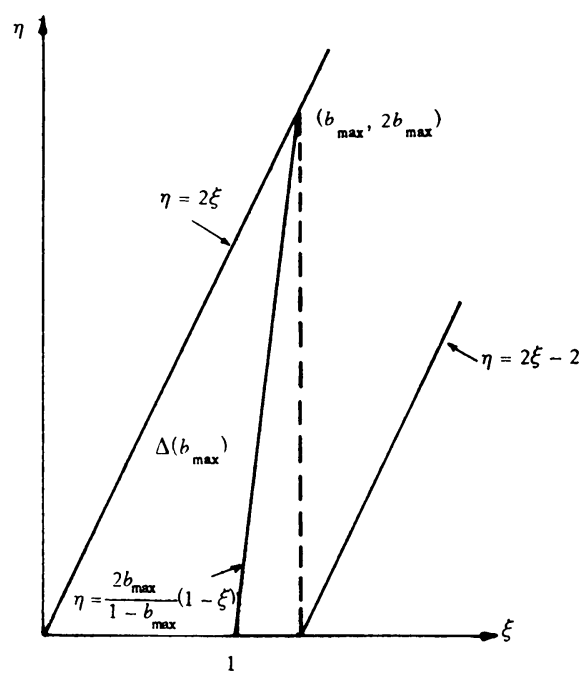

FIGURE 1. $\Delta\left(b_{\max }\right)$ for some $b_{\max }>1$.

tion, $R(b, \omega)$ takes the form 


$$
L(\xi, \eta)=1-\xi+2 \theta \xi+2 \theta\left(\xi-1-\theta \xi^{2}\right) \eta ;
$$

and now we are looking for the largest triangle $\Delta=\Delta\left(b_{\max }\right)$ in the $(\xi, \eta)$ plane with vertices $(0,0)(1,0)$ and $\left(b_{\max }, 2 b_{\max }\right)$ in which $L(\xi, \eta) \geqq 0$.

Now take $\theta \geqq 1 / 6$ and we shall prove (4). $L(\xi, \eta)$ is linear in $\eta$; hence, for every fixed $0 \leqq \xi_{0} \leqq \operatorname{Max}\left\{1, b_{\max }\right\}, \operatorname{Min}_{\Delta} L\left(\xi_{0}, \eta\right)$ is achieved in one of the two intersection points of the line $\xi=\xi_{0}$ with the boundary of $\Delta$, which consists of the lines $\eta=2 \xi$, $\eta=0$ and $\eta=\left[2 b_{\max } /\left(1-b_{\max }\right)\right](1-\xi)$. The possible intersection points are therefore, respectively, $\left(\xi_{0}, 2 \xi_{0}\right),\left(\xi_{0}, 0\right)$ and $\left(\xi_{0}, 2 b_{\max }\left(1-\xi_{0}\right) /\left(1-b_{\max }\right)\right)$. For $0 \leqq \xi_{0} \leqq$ $b_{\max }$, one of these points is $\left(\xi_{0}, 2 \xi_{0}\right)$ and

$$
L\left(\xi_{0}, 2 \xi_{0}\right)=\left(1-2 \theta \xi_{0}\right)\left(2 \theta \xi_{0}^{2}-\xi_{0}+1\right) .
$$

The quadratic term in (12) is positive for all $\theta>1 / 8$ and the linear term is nonnegative iff $\xi_{0} \leqq 1 / 2 \theta$. So, $L\left(\xi_{0}, 2 \xi_{0}\right) \geqq 0$ for $0 \leqq \xi_{0} \leqq b_{\max }$, iff $\xi_{0} \leqq 1 / 2 \theta$ and we can take at most $b_{\max }=1 / 2 \theta$. For $0 \leqq \xi_{0} \leqq 1$, one of the intersecting points is $\left(\xi_{0}, 0\right)$ and

$$
L\left(\xi_{0}, 0\right)=1-\xi_{0}+2 \theta \xi_{0} \geqq 0 .
$$

Finally, the last point mentioned above plays a role when (a) $b_{\max }<1$ and $b_{\max }<$ $\xi_{0}<1$ or (b) $b_{\max }>1$ and $1<\xi_{0}<b_{\max }$. In these cases, setting $b_{\max }=1 / 2 \theta$, we have

$$
\begin{aligned}
L\left(\xi_{0}, 2 b_{\max }\left(1-\xi_{0}\right) /\left(1-b_{\max }\right)\right) & \\
= & (1-2 \theta)^{-1}(1-2 \theta \xi)\left[2 \theta \xi^{2}-(1+2 \theta) \xi+(1+2 \theta)\right] .
\end{aligned}
$$

The quadratic term of Eq. (12b) is nonnegative for $\theta \geqq 1 / 6$; the linear term $(1-2 \theta \xi) /(1-2 \theta)$ is nonnegative iff $\xi_{0} \geqq 1 / 2 \theta$ in case (a) and $\xi_{0} \leqq 1 / 2 \theta$ in case (b), which agrees in both cases with our choice $b_{\max }=1 / 2 \theta$. This proves (4).

For $0<\theta<1 / 6$, it can be shown that the stability criterion is (9) with $1<b_{\max }=$ $b_{\max }(\theta)<3$, namely, that the $b_{\max }$ achieves the largest value at $\theta=1 / 6, b_{\max }(1 / 6)=3$.

For $\theta=0$, we go back to the Lax-Wendroff scheme and $b_{\max }=1$. In the following table, we give some values of the bound $b_{\max }(\theta), 0<\theta<1 / 6$.

\begin{tabular}{crrrrr}
\hline$\theta$ & .01 & .05 & .1 & .125 & .15 \\
\hline$b_{\max }(\theta)$ & 1.02 & 1.12 & 1.38 & 1.72 & 2.37 \\
\hline
\end{tabular}

At last, we show that the schemes are unconditionally unstable for $\theta<0$. For any $\Delta=\Delta\left(b_{\max }\right)$, take a point $(\xi, 0)$ with $\xi<1, \xi$ close enough to $1 .(\xi, 0) \in \Delta$ and by (12a) $L(\xi, 0)<0$, so, by continuity, $L<0$ in a neighborhood of this point and, by (8a), we have that (8) is not satisfied in all $\Delta$.

Department of Mathematical Sciences

Tel-Aviv University

Ramat-Aviv, Tel-Aviv, Israel

1. S. Abarbanel \& G. Zwas, "An iterative finite-difference method for hyperbolic systems," Math. Comp., v. 23, 1969, pp. 549-565. MR 40 \#1044.

2. S. ABARBANEL \& M. GoldBERG, "Numerical solution of quasi-conservative hyperbolic systems - the cylindrical shock problem," J. Computational Phys., v. 10, 1972, pp. 1-21.

3. P. D. LAX \& B. Wendroff, "Systems of conservation laws," Comm. Pure Appl. Math., v. 13, 1960, pp. 217-237. MR 22 \#11523. 